\title{
Participation in physical activity is associated with reduced nocturnal hypoxaemia in males
}

\author{
David Stevens ${ }^{1,2}$, Sarah Appleton ${ }^{1}$, Yohannes Melaku [1] ${ }^{1}$, Sean Martin², \\ Robert Adams ${ }^{1,4}$ and Gary Wittert ${ }^{3,5}$, on behalf of the MAILES investigators
}

Affiliations: 'Sleep Health, Flinders Health and Medical Research Institute, Flinders University, Adelaide, SA, Australia. ${ }^{2}$ Centre for Nutritional and Gastrointestinal Diseases, South Australian Health \& Medical Research Institute, Adelaide, SA, Australia. ${ }^{3}$ Adelaide Medical School, The University of Adelaide, Adelaide, SA Australia. ${ }^{4}$ Respiratory and Sleep Services, Southern Adelaide Local Health Network, SA, Adelaide, Australia. ${ }^{5}$ Freemasons Centre for Male Health and Health and Wellbeing. The University of Adelaide, and the South Australian Health and Medical Research Institute, SA, Australia.

Correspondence: David Stevens, Sleep Health, Flinders Health and Medical Research Institute, Flinders University, Bedford Park, Adelaide, South Australia 5001, Australia. E-mail: david.stevensaflinders.edu.au

ABSTRACT Moderate to vigorous physical activity (MVPA) interventions reduce the severity of obstructive sleep apnoea (OSA); however, little epidemiological research exists to confirm these findings.

789 participants from the population-based Men Androgen Inflammation Lifestyle Environment and Stress (MAILES) Study underwent polysomnography. MVPA was assessed using the Active Australia questionnaire, which was completed when participants were first recruited to the MAILES study (2002-2006), and again in 2010. Multinomial logistic regressions established odds ratio between OSA severity categories with MVPA, whilst adjusted linear models determined associations between OSA metrics with MVPA.

Cross-sectionally, each hour of MVPA was associated with reduced severity of mean oxygen desaturation (unstandardised $\beta(B)=-0.002, p=0.043$ ) and reduced time below $90 \%$ oxygen saturation $(\mathrm{B}=-0.03, \mathrm{p}=0.034)$.

Longitudinally, each hour increase in MVPA was associated with a $4 \%$ reduction in the odds of severe OSA and less severe mean oxygen desaturation $(B=-0.003, p=0.014)$, time below $90 \%$ oxygen saturation $(B=-0.02, p=0.02)$, and mean duration of apnoeas $(B=-0.004, p=0.016)$.

MVPA is associated with reduced hypoxaemia in a cohort of community dwelling males, approximately half of whom had untreated OSA. As nocturnal intermittent hypoxaemia is associated with cardiometabolic disorders, MVPA may offer protection for patients with OSA.

@ERSpublications

This study provides epidemiological evidence that moderate to vigorous physical activity is associated with less severe OSA-induced hypoxaemia. This result suggests that MVPA should be actively implemented in treatment regimens for people with OSA. https://bit.ly/3a9asiZ

Cite this article as: Stevens D, Appleton S, Melaku Y, et al. Participation in physical activity is associated with reduced nocturnal hypoxaemia in males. ERJ Open Res 2021; 7: 00852-2020 [https:// doi.org/10.1183/23120541.00852-2020].

This article has supplementary material available from openres.ersjournals.com

Received: 14 Nov 2020 | Accepted: 19 Jan 2021

Copyright $\odot$ The authors 2021. This version is distributed under the terms of the Creative Commons Attribution NonCommercial Licence 4.0. For commercial reproduction rights and permissions contact permissions@ersnet.org 


\section{Introduction}

Obstructive sleep apnoea (OSA) is the recurrent reduction (hypopnoea), or complete cessation (apnoea), of airflow during sleep, resulting in intermittent hypoxaemia and arousals from sleep. OSA severity is clinically assessed by the number of apnoeas and hypopnoeas that occur each hour (apnoea/hypopnoea index (AHI)). Severe OSA $(\mathrm{AHI} \geqslant 30)$, in particular is associated with increased risk of coronary heart disease and stroke [1], type 2 diabetes [2], and all-cause mortality [3,4]. Recent cohort studies indicate that, whilst $10 \%$ of the population have been diagnosed with OSA, roughly $50 \%$ of sample populations had undiagnosed OSA $[5,6]$, with $26-50 \%$ of men and up to $25 \%$ women experiencing moderate-to-severe OSA.

The current standard treatment for OSA is continuous positive airway pressure (CPAP). In the majority of patients with OSA, varying anatomical and physiological traits causes the upper airway to be collapsible above atmospheric pressure once conscious control of the upper airway is absent during sleep [7]. As the name implies, CPAP continuously delivers positively pressured air to prevent the upper airway from narrowing or collapsing.

Whilst CPAP is effective at reducing the AHI to near negligible levels, compliance, defined as at least $4 \mathrm{~h}$ of use per night for $70 \%$ of the nights, has historically been poor. RoTENBURG et al. [8] showed that for each year over a 20-year period (1994-2015), up to $40 \%$ of CPAP users were "non-compliant". Furthermore, recent large randomised controlled trials have demonstrated that CPAP does not reduce the chances of a secondary cardiovascular or cerebrovascular event [9], nor improve glycaemic control of type 2 diabetes [10].

In contrast, of moderate to vigorous intensity physical activity (MVPA) decreases the risk of secondary cardiovascular and cerebrovascular events [11, 12], as well as glycaemic control of type 2 diabetes [13]. Importantly, short-term MVPA intervention studies significantly reduce AHI (for reviews, see EDwARDs et al. [14] and MEDELSON et al. [15]). MVPA is effective at reducing BMI through reductions in adiposity, which is a modifiable risk factor for OSA [16], whereas CPAP has very little effect on BMI or weight distribution [17]. A recent cohort study showed more time spent undertaking MVPA was associated with decreased prevalence of self-reported diagnosis of OSA but could not provide data on OSA severity [18]. Likewise, another cohort study showed more time spent undertaking MVPA was associated with reduced odds of polysomnography measured moderate to severe OSA [19]. Their models, however, only adjusted for age and BMI, and no other known important determinants of OSA [19].

Therefore, the aim of this study was to determine the cross-sectional associations between MVPA and the presence and severity of OSA, and the associations between changes in MVPA over time and the presence and severity of OSA, whilst adjusting for known determinants of OSA.

\section{Materials and methods}

Participants

Data for this study was collected as part of the Men Androgen Inflammation Lifestyle Environment and Stress (MAILES) Study and has been described in detail previously [20]. Briefly, the MAILES cohort was a combination of two existing cohorts (the North West Adelaide Health Study, and the Florey Adelaide Male Aging Study). These cohorts consisted of randomly selected community-dwelling men living in Adelaide, SA, Australia (2002-2006), recruited using the same sampling frame and methodology. Recruitment occurred by random selection of phone numbers from the electronic telephone directory. Participants who were willing to be involved underwent computer-assisted telephone interviews, answered detailed questions regarding biographical, sociodemographic, co-morbidities and lifestyle risk factors. Participants also underwent a clinical assessment including anthropometry, sphygmomanometer and a fasting blood sample.

The study was approved by both the North West Adelaide Health Service (approval number 201005) and the Royal Adelaide Hospital institutional ethics committee (approval number 02305H). All participants gave written informed consent, with consent collected for each new data collection stage.

\section{Polysomnography}

In 2010, a computer assisted telephone interview $(n=1629)$ identified 1445 participants without a previous diagnosis of OSA by polysomnography (PSG). These men were invited by to participate in an in-home PSG (Embletta X100; Embla Systems, Thornton, CO, USA) with 1087 (75.2\%) agreeing. By the conclusion of the study period, 861 PSGs had been attempted, including repeated PSGs due to initial failure.

The PSG measured electroencephalography (EEG), electrooculography, chin electromyography, nasal pressure, thoracic and abdominal effort, peripheral pulse oximetry and body position. Trained staff visited study participants in their homes to set-up the sleep study, as well as record height and weight measurements. A single experienced PSG technician, who was blinded to all other survey and biomedical 
data, performed manual scoring of PSGs according to 2007 American Academy of Sleep Medicine (alternate) criteria [21], which is recommended for use in prospective epidemiological studies. Studies were considered acceptable with $3.5 \mathrm{~h}$ of sleep and $5.5 \mathrm{~h}$ of total recorded study time. Hypopnoeas were a $50 \%$ or more reduction in airflow coupled with either: 1) at least a 3\% oxygen desaturation, or 2) an EEG arousal. Apnoeas were a 10-s cessation of airflow. OSA severity was categorised as: mild (AHI 10-19 events $\cdot h^{-1}$ ), moderate (AHI 20-29 events $h^{-1}$ ) and severe (AHI $>30$ events $\cdot h^{-1}$ ). The cut-offs for classification were chosen because RUEHLAND et al. [22] showed that an AHI of 5 events $\cdot h^{-1}$ used to define sleep disordered breathing scored by the "recommended" American Academy of Sleep Medicine (AASM) criteria is equivalent to an AHI of 10 events $\cdot \mathrm{h}^{-1}$ of sleep using the alternate AASM definition.

The PSG provided the following OSA variables; AHI (events. $h^{-1}$ ), mean duration of apnoeas and hypopnoeas (seconds), AHI during rapid eye movement sleep (REM AHI), mean oxygen desaturation (\%), and percent of time spent below $90 \%$ oxygen saturation $\left(\mathrm{T}_{90 \%}\right)$. Participants were removed if their oxygen saturation was deemed of poor quality, determined by either; having an AHI $<10$ events.h ${ }^{-1}$ but had $>20 \%$ of the night below $\mathrm{T}_{90 \%}$, or spent more than $20 \%$ of the night below $\mathrm{T}_{90 \%}$, yet had no time below $80 \%$ oxygen saturation.

\section{Physical activity questionnaire}

Physical activity was measured by the Active Australia Survey [23]. During initial recruitment (2002-2006), participants completed the 1999 version of the questionnaire (then called the National Physical Activity Survey), which asked for the amount of time spent, in minutes, undertaking walking, moderated physical activity, and vigorous physical activity, over the past two weeks [24]. In 2010, participants completed an updated version of the questionnaire [23], which assessed activity over the previous week instead of the past two weeks (herein referred to as MVPA-2010).

To synthesise the results from both questionnaires, the values recorded during baseline by the National Physical Activity Survey were halved. Furthermore, vigorous physical activity levels in both questionnaires was given twice the weight of both walking and moderate vigorous physical activity, as per guidelines for both versions of the questionnaire [23, 24]. Thus, the MVPA levels for both questionnaires was the addition of the adjusted vigorous values with the unadjusted moderate and walking values. Longitudinal changes in MVPA levels were calculated by subtracting the baseline MVPA (2002-2006) from the 2010 MVPA assessment (herein referred to as MVPA- $\Delta$ ).

To increase interpretability of the MVPA values, both MVPA-2010 and MVPA- $\Delta$ were converted to hours per week. Participants who reported more than $56 \mathrm{~h} \cdot \mathrm{week}^{-1}$ for the MVPA-2010 (i.e. more than $8 \mathrm{~h} \cdot \mathrm{day}^{-1}$, which allows those in active professions, such as the construction industry, to be included in the analysis), or more than $56 \mathrm{~h} \cdot \mathrm{week}^{-1}$ of change in MVPA- $\Delta$, were excluded, as these values were considered unrealistic, suggesting the participant answered the question incorrectly.

\section{Statistical analysis}

Multinomial logistic regressions and linear models were used in this analysis, both of which adjusted for confounders. Confounders were determined by examining known or suspected determinants of both AHI and $\mathrm{T}_{90 \%}$ in univariate models. Covariates with a $\mathrm{p}<0.1$ for both $\mathrm{AHI}$ and $\mathrm{T}_{90 \%}$ were included in all analyses. The following variables were included as confounders: age, BMI, study group, income, testosterone (log transformed), inflammation using C-reactive protein (log-transformed), diabetes, cardiovascular disease, hypertension and smoking status. Additionally, for MVPA- $\Delta$, we also adjusted for baseline MVPA levels in hours. Details on collection methods of the known or suspected confounders, as well as results for the univariate analyses, are in the online supplementary material (Supplement S1).

Multinomial logistic regression analysis was used to determine the likelihood of the dependent variable, the OSA severity category associated with MVPA-2010 and MVPA- $\Delta$. For the linear regression models, the continuous dependent variables were AHI, REM AHI, mean oxygen desaturation, $\mathrm{T}_{90 \%}$ and mean duration of apnoeas and hypopnoeas. These metrics were all normalised by log transformation, however, to increase interpretability of the level of change in OSA/hypoxaemia metric associated with an hour change in MVPA-2010 and MVPA- $\Delta$, the regression coefficients were exponentially transformed. A further analysis is presented in the supplementary material (Supplement S2), which examines differences in OSA indices between participants who increased, and those who decreased, MVPA by at least five hours (300 $\mathrm{min}$ ) between baseline and 2010.

All statistical analyses were calculated in $\mathrm{R}$ (v3.1.0, R Foundation for Statistical Computing, Vienna, Austria), and utilised the "LSR" and "NNET" packages. All models were assessed for linearity and homoscedasticity (residual versus fitted plots and scale-location plots), normal distribution of residuals (Q-Q plot), and influential values (Cooks $\mathrm{D}>0.5$ ). A p-value $<0.05$ was considered significant. Figures were created in Prism (GraphPad Software, San Diego, CA, USA). 


\section{Results}

Participants

Figure 1 illustrates the derivation of the final analysis set; 34 participants were excluded due to inadequate OSA data, whilst an additional 25 participants were removed from the $\mathrm{T}_{90 \%}$ analysis due to potential signal quality issues. Nine participants were excluded for reporting more than $56 \mathrm{~h} \cdot \mathrm{week}^{-1}$ of MVPA, whilst a further four were excluded for reporting a change or more than $56 \mathrm{~h} \cdot \mathrm{week}^{-1}$. This resulted in the analysis of 789 participants, whose characteristics are outlined in table 1. One participant was excluded from the analysis for mean oxygen desaturation due to be an influential value according to the Cook's D of 0.5 .

\section{Associations between MVPA-2010 with OSA severity categories and nocturnal hypoxaemia} metrics

Multinomial logistic regressions indicated that each hour of MVPA was associated with modest but nonsignificant reduced odds for both mild OSA (OR 0.976, 95\% CI 0.95-1.01; $\mathrm{p}=0.11$ ) and severe OSA $(\mathrm{OR}=0.96,95 \%$ CI $0.91-1.01 ; \mathrm{p}=0.09)$, and a small, nonsignificant increased odds of moderate OSA $(\mathrm{OR}=1.01,95 \%$ CI $0.98-1.04 ; \mathrm{p}=0.54)$.

Forest plots derived from adjusted linear regressions examining cross-sectional associations between MVPA and OSA metrics are shown in figure 2. More time spent undertaking MVPA-2010 was associated with reduced mean oxygen desaturation (unstandardised $\beta(B)=-0.002, p=0.043$ ) and reduced $\mathrm{T}_{90 \%}$ $(B=-0.03, p=0.034)$. No significant associations occurred with AHI $(b=-0.01, p=0.15)$, REM AHI $(\mathrm{B}=-0.005, \mathrm{p}=0.59)$, or the duration of apnoeas $(\mathrm{B}=-0.001, \mathrm{p}=0.38)$ and hypopnoeas $(\mathrm{B}=-0.001$, $\mathrm{p}=0.58)$.

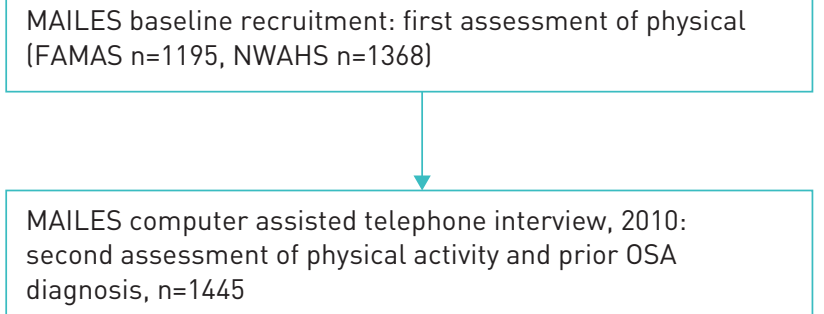

Participants with valid MVPA data, $\mathrm{n}=789$

FIGURE 1 Consort diagram showing number, and reasons, for exclusion from analysis. MAILES: Men Androgen Inflammation Lifestyle Environment and Stress; FAMAS: Florey Adelaide Men's Aging Study; NWAHS: North West Adelaide Health Study; OSA: obstructive sleep apnea; MVPA: moderate to vigorous physical activity. 


\section{TABLE 1 Polysomnographic characteristics of study participants}

Age years

Body mass index $\mathrm{kg} \cdot \mathrm{m}^{-2}$

AHI events. $h^{-1}$

REM AHI events $\cdot h^{-1}$

Mean oxygen desaturation \%

$\mathrm{T}_{90 \%} \%$

Mean hypopnoea duration s

Mean apnoea duration s

MVPA-2010 h

MVPA- $\Delta$ h

Data are presented as median \pm interquartile range. AHI: apnoea/hypopnoea index; REM: rapid eye movement; T90\%: amount of sleep with oxygen saturation $<90 \%$; MVPA: moderate to vigorous physical activity; MVPA-2010: time spent undertaking MVPA during 2010 collection; MVPA- $\Delta$ : change in time spent undertaking MVPA from baseline (2002-2006) to 2010.

A sensitivity analysis (not included) indicates that when participants with pulse oximetry irregularities were included in the linear regressions, no differences in associations between MVPA-2010 and OSA indices occurred.

\section{Associations between MVPA- $\triangle$ with OSA severity categories and nocturnal hypoxaemia metrics}

Multinomial logistic regressions indicated that each hour increase in MVPA- $\Delta$ was associated with a small, nonsignificant reduced odds of mild OSA (OR 0.980, 95\% CI 0.96-1.01; p=0.12), no change in odds of moderate OSA (OR 1.00, 95\% CI 0.97-1.03; $\mathrm{p}=0.89$ ) and a moderate, significant reduced odds of severe OSA (OR 0.960, 95\% CI 0.93-0.99; p=0.03).

Forest plots derived from adjusted linear regressions examining associations between MVPA- $\Delta$ and OSA metrics are shown in figure 3 . There were significant inverse associations between MVPA- $\Delta$ and mean oxygen desaturation $(\mathrm{B}=-0.003, \mathrm{p}=0.014), \mathrm{T}_{90 \%}(\mathrm{~B}=-0.02, \mathrm{p}=0.02)$, and mean duration of apnoeas $(B=-0.004, p=0.016)$. No significant associations occurred with AHI $(B=-0.008, p=0.17)$, REM AHI $(B=0.001, p=0.88)$ or duration of hypopneas $(B=-0.001, p=0.27)$. For interpretability, the change in each metric per hour of MVPA-2010 is shown in table 2.

A sensitivity analysis (not included) indicates that when participants with pulse oximetry irregularities were included in the linear regressions, $\mathrm{T}_{90 \%}$ was no longer associated with MVPA- $\Delta(\mathrm{B}=-0.015, \mathrm{p}=0.07)$. No other changes in associations occurred.

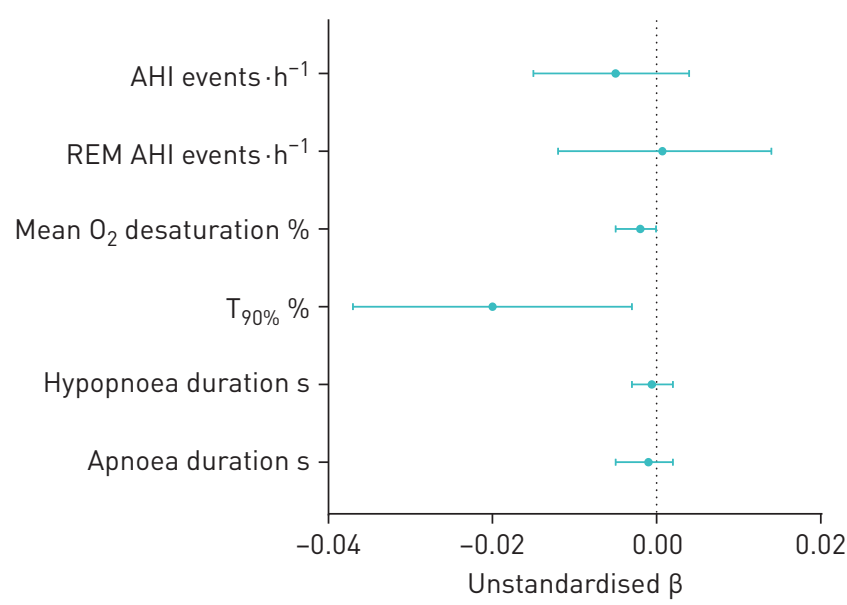

FIGURE 2 Cross-sectional associations between moderate to vigorous physical activity (MVPA) in 2010 and obstructive sleep apnea metrics. X-axis indicates log-transformed unstandardised $\beta$. Adjustments: age, body mass index, study group (Florey Adelaide Men's Aging Study versus North West Adelaide Health Study). income, serum testosterone, serum C-reactive protein, cardiovascular disease status, diabetes status, hypertension status, Missing data: MVPA difference: 36; income: 20; testosterone: 66; inflammation: 65; diabetes status: 12 ; cardiovascular disease status: 14 ; blood pressure: 26 ; mean $\mathrm{O}_{2}$ desaturation: 1 . $\mathrm{AHI}$ apnoea/hypopnoea index; REM: rapid eye movement; $T_{90 \%}$ : amount of sleep with oxygen saturation $<90 \%$. 


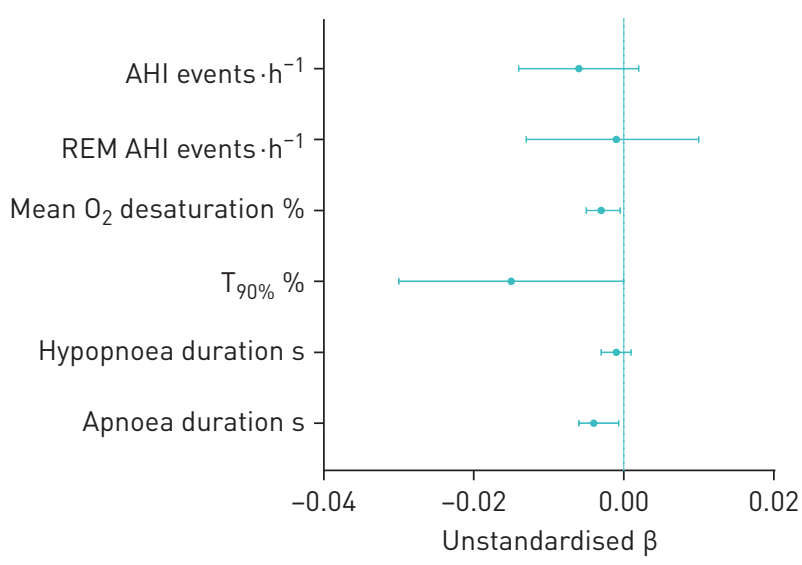

FIGURE 3 Cross-sectional associations between changes in moderate to vigorous physical activity (MVPA) from baseline (2002-2006) to 2010 (MVPA- $\Delta$ ) and obstructive sleep apnea metrics. X-axis indicates log-transformed unstandardised $\beta$. Adjustments: age, body mass index, study group (Florey Adelaide Men's Aging Study versus North West Adelaide Health Study), income, serum testosterone, serum C-reactive protein, cardiovascular disease status, diabetes status, hypertension status Missing data: MVPA: 35; income: 20; testosterone: 66 ; inflammation: 65; diabetes status: 12; cardiovascular disease status: 14 ; blood pressure: 26; mean $\mathrm{O}_{2}$ desaturation: 1. AHI: apnoea/hypopnoea index; REM: rapid eye movement; $\mathrm{T}_{90 \%}$ : amount of sleep with oxygen saturation $<90 \%$.

\section{Discussion}

This study highlights spending more time engaged in MVPA was associated with less severe nocturnal hypoxaemia indices, but in cross-sectional analyses was not associated with AHI or REM AHI. The same associations with hypoxaemia indices persisted when we examined the changes in time spent engaging in MVPA from baseline (2002-2006) to 2010, suggesting this is a robust finding. Furthermore, increasing MVPA over time was associated with significantly reduced odds of severe OSA.

The results of this study are consistent with the cross-sectional data of DA SILVA et al. [19], despite this study utilising more stringent adjustments for known confounders of OSA, and the different physical activity questionnaires used. Importantly, to our knowledge, this is the first study to examine associations of longitudinal changes in MVPA on OSA metrics. Thus, this study adds further evidence highlighting the potential benefits of MVPA in reducing the severity of OSA [14, 15].

A novel finding of this study was the significant association between increasing the amount of time undertaking MVPA between baseline and 2010 and shorter mean duration of apnoeas, along with the nonsignificant association with shorter mean duration of hypopnoeas. This is clinically important, as longer duration of apnoeas and hypopnoeas are associated with increased prevalence and severity of hypertension [25-27], and increased prevalence of cardiovascular disease [28]. The reductions in apnoea duration, but not hypopnoea duration, may provide a mechanism as to why increased MVPA over time may improve hypoxaemia. Yet, as MVPA-2010 was not associated with either apnoea or hypopnoea mean duration but was associated with improved hypoxaemia indices, changed in MVPA must affect other contributors to OSA and hypoxaemia severity.

TABLE 2 Percentage change in obstructive sleep apnoea (OSA) metrics for each hour increase in MVPA-2010 and MVPA- $\Delta$

\begin{tabular}{lcc} 
OSA index & MVPA-2010 & MVPA- $\Delta$ \\
\hline AHI events $\cdot h^{-1}$ & -0.5 & -0.6 \\
${\text { REM AHI events } \cdot h^{-1}}^{\text {Mean oxygen desaturation \% }}$ & -0.1 & -0.1 \\
T90\% $\%$ & $-0.2^{\#}$ & $-0.3^{\#}$ \\
Mean hypopnoea duration s & $-2.0^{\#}$ & $-1.5^{\#}$ \\
Mean apnoea duration s & -0.06 & -0.1 \\
\hline
\end{tabular}

MVPA-2010: time spent undertaking moderate to vigorous physical activity (MVPA) during 2010 collection; MVPA- $\Delta$ : change in time spent undertaking MVPA from baseline (2002-2006) to 2010; AHI: apnoea/ hypopnoea index; REM: rapid eye movement; $\mathrm{T}_{90 \%}$ : amount of sleep with oxygen saturation $<90 \%$. ${ }^{\#}$ : significant association. 
Anatomically, MVPA reduces the fat mass around the upper airway and in the tongue, which has been shown to improve symptoms of OSA $[29,30]$. In addition, increased MVPA may target non-anatomical contributors of OSA. For example, the upper airway muscles of patients with OSA fatigue easily, meaning people with OSA are predisposed to experiencing narrowing and collapsing of the upper airway during sleep [31]. In adults without OSA, MVPA results in upper airway muscles that are less susceptible to fatigue [32, 33]. Another non-anatomical contributor is unstable respiratory control, where a person experiences cycles of hyperventilation and hypoventilation due to small changes in blood gasses. Adults who regularly undertake MVPA show more stable respiratory control [34]. Importantly, similar results have been shown in adults with congestive heart failure, a condition increases a person's susceptibility to unstable respiratory control [35]. As these potential mechanisms have not been examined, however, it remains uncertain why undertaking MVPA is associated with reduced odds of severe OSA and less severe nocturnal hypoxaemia indices, and therefore should be the focus of future research.

Although the changes in hypoxaemia indices per hour of MPVA in this study were not large, epidemiological studies examining other heath conditions have shown small improvements convey large protective benefits at a population level. For example, a $2-\mathrm{mmHg}$ reduction in systolic blood pressure reduced the risk of death due to ischaemic heart disease and stroke by $7 \%$ and $10 \%$ respectively [36]. Similarly, in the UK, a $1 \%$ improvement in $\mathrm{HbAl}_{\mathrm{c}}$ resulted in approximately GBP $2.5 \mathrm{bn}$ reduction over 25 years in healthcare costs due to complications from diabetes [37].

The strength of this study lies in the clinical polysomnography collected in a large cohort of participants representative of the urban dwelling population. A further strength of this study is longitudinal nature of the MVPA data, with the Active Australia questionnaires already used extensively in other epidemiological studies $[38,39]$.

Only examining the male population can be considered a limitation of this study, as the findings cannot be applied to women. A further limitation is the lack of objectively measured MVPA data; however, as large scale actigraphy measurement was unfeasible until the mid-2010s, this was unavoidable.

As there has only been small, short-duration studies examining the effect of MVPA interventions on OSA, future large randomised, controlled trials are needed to determine the effect of undertaking MVPA without CPAP, and the combination of both MVPA and CPAP, has on OSA severity indices. Additionally, future studies should examine potential mechanisms by which MPVA improves hypoxaemia indices. To do this, longitudinal changes in OSA metrics, as well as studies that can directly measure the anatomical and non-anatomical contributors to OSA, are needed. Importantly, all future studies should objectively measure MVPA by actigraphy, as well as examine all genders.

Our study provides evidence more time spent undertaking MVPA was associated with lower odds of severe OSA, and less severe hypoxaemia. Importantly, whilst CPAP is effective at eliminating hypoxaemia [40], cardiovascular and metabolic health problems still remain in patients with OSA $[9,10]$. Therefore, the results of this study, when coupled with the well-established benefits of MVPA on other health conditions [11-13], suggest that MVPA should be prescribed for all patients with OSA.

Acknowledgements: We thank Andrew Vincent (University of Adelaide) for guidance with the statistical analysis.

Data sharing: De-identified participant data used in this analysis can be shared upon reasonable request. Please contact the corresponding author.

Author contributions: D. Stevens conceived the overall analysis. D. Stevens and Y. Melaku planned the analysis, whilst D. Stevens carried out the analysis. S. Appleton, S. Martin, R. Adams and G. Wittert are key investigators on the MAILES study. All authors contributed to the interpretation and analysis, as well as the final manuscript. D. Stevens takes full responsibility for the integrity of the data and accuracy of the analysis results.

Conflict of interest: D. Stevens has nothing to disclose. S. Appleton has nothing to disclose. Y. Melaku has nothing to disclose. S. Martin has nothing to disclose. R. Adams reports grants from the National Health and Medical Research Council, the ResMed Foundation, The Hospital Research Foundation, The Queen Elizabeth Hospital, and the Freemason's Foundation for Men's Health, during the conduct of the study. G. Wittert reports grants from National Health and Medical Research Council, the ResMed Foundation, The Hospital Research Foundation, The Queen Elizabeth Hospital, and the Freemason's Foundation for Men's Health, during the conduct of the study.

Support statement: There was no funding specifically for this analysis. The MAILES Study has been supported by the National Health and Medical Research Foundation (APP 627227, Australia), the Hospital Research Foundation (Australia), and the ResMed Foundation (USA). Funding information for this article has been deposited with the Crossref Funder Registry.

\section{References}

1 Loke YK, Brown JW, Kwok CS, et al. Association of obstructive sleep apnea with risk of serious cardiovascular events: a systematic review and meta-analysis. Circ Cardiovasc Qual Outcomes 2012; 5: 720-728. 
2 Drager LF, Togeiro SM, Polotsky VY, et al. Obstructive sleep apnea: a cardiometabolic risk in obesity and the metabolic syndrome. J Am Coll Cardiol 2013; 62: 569-576.

3 Kendzerska T, Mollayeva T, Gershon AS, et al. Untreated obstructive sleep apnea and the risk for serious long-term adverse outcomes: a systematic review. Sleep Med Rev 2014; 18: 49-59.

4 Xie C, Zhu R, Tian Y, et al. Association of obstructive sleep apnoea with the risk of vascular outcomes and all-cause mortality: a meta-analysis. BMJ Open 2017; 7: e013983.

5 Adams R, Appleton S, Taylor A, et al. Are the ICSD-3 criteria for sleep apnoea syndrome too inclusive? Lancet Respir Med 2016; 4: e19-e20.

6 Heinzer R, Vat S, Marques-Vidal P, et al. Prevalence of sleep-disordered breathing in the general population: the HypnoLaus study. Lancet Respir Med 2015; 3: 310-318.

7 Kirkness JP, Peterson LA, Squier SB, et al. Performance characteristics of upper airway critical collapsing pressure measurements during sleep. Sleep 2011; 34: 459-467.

8 Rotenberg BW, Murariu D, Pang KP. Trends in CPAP adherence over twenty years of data collection: a flattened curve. J Otolaryngol Head Neck Surg 2016; 45: 1-9.

9 Sanchez-de-la-Torre M, Sanchez-de-la-Torre A, Bertran S, et al. Effect of obstructive sleep apnoea and its treatment with continuous positive airway pressure on the prevalence of cardiovascular events in patients with acute coronary syndrome (ISAACC study): a randomised controlled trial. Lancet Respir Med 2019; 8: 359-367.

10 Loffler KA, Heeley E, Freed R, et al. Continuous positive airway pressure treatment, glycemia, and diabetes risk in obstructive sleep apnea and comorbid cardiovascular disease. Diabetes Care 2020; 43: 1859-1867.

11 Darden D, Richardson C, Jackson EA. Physical activity and exercise for secondary prevention among patients with cardiovascular disease. Curr Cardiovasc Risk Rep 2013; 7: 411-416.

12 Winzer EB, Woitek F, Linke A. Physical activity in the prevention and treatment of coronary artery disease. J Am Heart Assoc 2018; 7: e007725.

13 Colberg SR, Sigal RJ, Fernhall B, et al. Exercise and type 2 diabetes: the American College of Sports Medicine and the American Diabetes Association: joint position statement. Diabetes Care 2010; 33: el47-e167.

14 Edwards BA, Bristow C, O'Driscoll DM, et al. Assessing the impact of diet, exercise and the combination of the two as a treatment for OSA: a systematic review and meta-analysis. Respirology 2019; 24: 740-751.

15 Mendelson M, Bailly S, Marillier M, et al. Obstructive sleep apnea syndrome, objectively measured physical activity and exercise training interventions: a systematic review and meta-analysis. Front Neurol 2018; 9:73.

16 Young T, Peppard PE, Gottlieb DJ. Epidemiology of obstructive sleep apnea: a population health perspective. Am J Respir Crit Care Med 2002; 165: 1217-1239.

17 Ou Q, Chen B, Loffler KA, et al. The effects of long-term CPAP on weight change in patients with comorbid OSA and cardiovascular disease: data from the SAVE trial. Chest 2019; 155: 720-729.

18 Hall KA, Singh M, Mukherjee S, et al. Physical activity is associated with reduced prevalence of self-reported obstructive sleep apnea in a large, general population cohort study. J Clin Sleep Med 2020; 16: 1179-1187.

19 da Silva RP, Martinez D, Pedroso MM, et al. Exercise, occupational activity, and risk of sleep apnea: a cross-sectional study. J Clin Sleep Med 2017; 13: 197-204.

20 Grant JF, Martin SA, Taylor AW, et al. Cohort profile: The men androgen inflammation lifestyle environment and stress (MAILES) study. Int J Epidemiol 2014; 43: 1040-1053.

21 Iber C, Ancoli-Israel S, Chesson AL, et al. The AASM manual for the scoring of sleep and associated events: rules, terminology and technical specifications. Westchester, American Academy of Sleep Medicine, 2007.

22 Ruehland WR, Rochford PD, O'Donoghue FJ, et al. The new AASM criteria for scoring hypopneas: impact on the apnea hypopnea index. Sleep 2009; 32: 150-157.

23 The Active Australia Survey: a guide and manual for implementation, analysis, and reporting. Canberra, Australian Institute for Health and Welfare (AIHW), 2003.

24 Armstrong T, Bauman A, Davies J. Physical activity patterns of Australian adults. Results of the 1999 National Physical Activity Survey. Canberra, Australia, Australian Institute for Health and Welfare, 2000.

25 André S, Andreozzi F, Van Overstraeten C, et al. Cardiometabolic comorbidities in obstructive sleep apnea patients are related to disease severity, nocturnal hypoxemia, and decreased sleep quality. Respir Res 2020; 21: 35 .

$26 \mathrm{Wu} \mathrm{H}$, Zhan X, Zhao M, et al. Mean apnea-hypopnea duration (but not apnea-hypopnea index) is associated with worse hypertension in patients with obstructive sleep apnea. Medicine (Baltimore) 2016; 95: e5493.

27 Saraç S, Afsar GC. Effect of mean apnea-hypopnea duration in patients with obstructive sleep apnea on clinical and polysomnography parameter. Sleep Breath 2020; 24: 77-81.

28 Turhan M, Bostanci A, Bozkurt S. Estimation of cardiovascular disease from polysomnographic parameters in sleep-disordered breathing. Eur Arch Otorhinolaryngol 2016; 273: 4585-4593.

29 Mitchell LJ, Davidson ZE, Bonham M, et al. Weight loss from lifestyle interventions and severity of sleep apnoea: a systematic review and meta-analysis. Sleep Med 2014; 15: 1173-1183.

30 Wang SH, Keenan BT, Wiemken A, et al. Effect of weight loss on upper airway anatomy and the apnea hypopnea index: the importance of tongue fat. Am J Respir Crit Care Med 2020; 201: 718-727.

31 Kimoff RJ. Upperairway myopathy is important in the pathophysiology of obstructive sleep apnea. J Clin Sleep Med 2007; 3: 567-569.

32 VanRavenhorst-Bell HA, Coufal KL, Patterson JA, et al. A comparative study: tongue muscle performance in weightlifters and runners. Physiol Rep 2018; 6: e13923.

33 VanRavenhorst-Bell HA, Mefferd AS, Coufal KL, et al. Tongue strength and endurance: comparison in active and non-active young and older adults. Int J Speech Lang Pathol 2017; 19: 77-86.

34 McConnell AK, Semple ES. Ventilatory sensitivity to carbon dioxide: the influence of exercise and athleticism. Med Sci Sports Exerc 1996; 28: 685-691.

35 Tomita T, Takaki H, Hara Y, et al. Attenuation of hypercapnic carbon dioxide chemosensitivity after postinfarction exercise training: possible contribution to the improvement in exercise hyperventilation. Heart 2003 89: 404-410.

36 Lewington S, Clarke R, Qizilbash $\mathrm{N}$, et al. Age-specific relevance of usual blood pressure to vascular mortality: a meta-analysis of individual data for one million adults in 61 prospective studies. Lancet 2002; 360: 1903-1913. 
37 Baxter M, Hudson R, Mahon J, et al. Estimating the impact of better management of glycaemic control in adults with Type 1 and Type 2 diabetes on the number of clinical complications and the associated financial benefit. Diabet Med 2016; 33: 1575-1581.

38 Brown WJ, Burton NW, Marshall AL, et al. Reliability and validity of a modified self-administered version of the Active Australia physical activity survey in a sample of mid-age women. Aust N Z J Public Health 2008; 32: 535-541.

39 Brown WJ, McLaughlin D, Leung J, et al. Physical activity and all-cause mortality in older women and men. $\mathrm{Br} \mathrm{J}$ Sports Med 2012; 46: 664-668.

40 Sutherland K, Kairaitis K, Yee BJ, et al. From CPAP to tailored therapy for obstructive sleep Apnoea. Multidiscip Respir Med 2018; 13: 44. 